\title{
Quality Issues Identified During the Evaluation of Biosimilars by the European Medicines Agency's Committee for Medicinal Products for Human Use
}

\author{
Mark Cilia, ${ }^{1}$ Sol Ruiz, ${ }^{2,3}$ Peter Richardson, ${ }^{3}$ Tomas Salmonson, ${ }^{3,4}$ Anthony Serracino-Inglott, ${ }^{1,5}$ \\ Francesca Wirth, ${ }^{5}$ and John Joseph Borg ${ }^{1,3,6,7}$ (D)
}

Received 30 June 2017; accepted 24 September 2017; published online 12 October 2017

\begin{abstract}
The aim of this study was to identify trends in deficiencies raised during the EU evaluation of the quality part of dossiers for marketing authorisation applications of biosimilar medicinal products. All adopted day 120 list of questions on the quality module of 22 marketing authorisation applications for biosimilars submitted to the European Medicines Agency and concluded by the end of October 2015 was analysed. Frequencies of common deficiencies identified were calculated and summarised descriptions included. Frequencies and trends on quality deficiencies were recorded and presented for 22 biosimilar applications. Thirty-two 'major objections' for 9 products were identified from 14 marketing authorisation applications with 15 raised for drug substance and 17 for drug product. In addition, 547 'other concerns' for drug substance and 495 for drug product were also adopted. The frequencies and trends of the identified deficiencies together with their impact were discussed from a regulatory perspective and how these impact key manufacturing processes and key materials used in the production of biosimilars. This study provides an insight to the regulatory challenges prospective companies need to consider when developing biosimilars; it also helps elucidate common pitfalls in the development and production of biosimilars and in the submission of dossiers for their marketing authorisations. The results are expected to be of interest to pharmaceutical companies but also to regulators to obtain consistent information on medicinal products based on transparent rules safeguarding the necessary pharmaceutical quality of medicinal products.
\end{abstract}

KEY WORDS: biosimilars; deficiencies; marketing authorisations; pharmaceutical development; chemistry manufacturing control.

\section{INTRODUCTION}

For an innovative medicinal product falling within the scope of the centralised procedure (1) to be placed on the European Union (EU)/European Economic Area (EEA) market, a marketing authorisation must be obtained from the European Commission (EC) following successful assessment

\footnotetext{
${ }^{1}$ Medicines Authority, Sir Temi Zammit Buildings, Malta Life Sciences Park, San Ġwann, SGN 3000, Malta.

${ }^{2}$ Agencia Española de Medicamentos y Productos Sanitarios, Parque Empresarial Las Mercedes, Edificio 8, C/Campezo 1, 28022, Madrid, Spain.

${ }^{3}$ European Medicines Agency, 30 Churchill Place, Canary Wharf, London, E14 5EU, UK.

${ }^{4}$ Läkemedelsverket, Dag Hammarskjölds väg 42, 75237, Uppsala, Sweden.

${ }^{5}$ Department of Pharmacy, University of Malta, Msida, Malta.

${ }^{6}$ Pharmacy Department, Faculty of Biology, University of Tor Vergata, Rome, Italy.

${ }^{7}$ To whom correspondence should be addressed. (e-mail: johnjoseph.borg@gov.mt)
}

of a dossier submitted through the centralised procedure to the European Medicines Agency (EMA). The marketing authorisation is granted by the EC according to a scientific opinion from the EMA's Committee for Medicinal Products for Human Use (CHMP) after undertaking (through its rapporteur and co-rapporteur teams) a scientific evaluation of the dossier submitted by the applicant in the form of a Common Technical Document-CTD (2). During this evaluation, specifically at day 120 of the procedure, the CHMP adopts a list of concerns related to quality, safety and efficacy, which are forwarded to the applicant to be addressed and resolved prior to the CHMP adopting an opinion on the benefitrisk profile of the medicinal product (3).

Biosimilar medicinal products, as defined in EU legislation, when they fulfil the mandatory criteria fall within the scope of this centralised procedure (1). A biosimilar can be defined as a biological product that has been demonstrated to be similar to a biological reference product, which is normally the originator biological product $(4,5)$. Biosimilarity means that the biological product is highly similar to the reference 
product despite minor differences in components such that there are no clinically meaningful differences between the biological and reference product in terms of safety, purity and potency of the product (4). In the EU, the applicant is required to submit a full module 3 of the CTD on the quality part as for all biological products together with data following a comparability study carried out between the biosimilar and its reference product in line with EU quality guidelines $(6,7)$.

In this study, all CHMP-adopted day 120 list of questions on the quality assessment part of Marketing Authorisation Applications (MAAs) submitted for biosimilar products and which was concluded by October $(n=22) 2015$ (8) was reviewed to identify common quality issues and to assess the frequency of identified deficiencies. The list of day 120 questions was used as this constitutes conclusions of the first consolidated scientific review of the data submitted by the prospective applicant endorsed by the CHMP. The quality questions raised during these assessments are included and may be in edited form to maintain an appropriate level of confidentiality, while ensuring the highest possible level of transparency.

The results show that the majority of submitted MAAs for biosimilars contained reports and documents that lacked data or clarity in a systematic way, considered by the CHMP as necessary to adequately understand the manufacturing processes involved for both drug substance and drug product. The impact of the frequencies and trends in quality concerns identified during the assessment of the CHMP is discussed from a regulatory perspective. The results of this study are expected to be of interest to pharmaceutical companies developing biosimilars and to regulators to be able to obtain consistent information on medicinal products based on transparent rules safeguarding the necessary pharmaceutical quality of medicinal products. It should be noted that the authorisations from which this report is compiled span a tenyear period and some guideline requirements have changed over this period. Therefore, any experience cited which could affect current biosimilar development plans should be considered in light of current EMA guidelines.

\section{MATERIALS AND METHODS}

\section{Creation and Analysis of a Database on Quality Concerns for Biosimilars}

All adopted CHMP day 120 questions for 22 MAAs of biosimilars concluded by October 2015 (8) were used to create a database of quality-related deficiencies. The number and type of biosimilar MAAs reviewed are included in Table I. Some applications were duplicate applications and hence had the same concerns identified by the CHMP. For these applications, since the questions were exactly identical, they were considered only once in the results and the statistical analysis.

The numbers and types of objections adopted by CHMP on module 3 of the CTD were arranged and sorted according to the CTD sections for drug substance and drug product (9). The deficiencies are termed by CHMP as either 'major objections' or 'other concerns' depending on their severity and impact on quality.

As part of the assessment procedure, CHMP adopts the terminology 'major objection' for serious deficiencies which must be resolved by the applicant prior to a positive CHMP opinion for a marketing authorisation (MA) and which if remain unresolved would lead to rejection of the MAA. The CHMP adopts the terminology 'other concerns' for those deficiencies which though they must be resolved prior to the issue of the MA, measures can be put in place post authorisation upon commitment. The module 3 sections and subsections used for classification of these concerns for drug substance and drug product are listed in Tables II and III, respectively.

Questions were reviewed and the absolute frequency with which a concern was listed per section and subsection of the CTD was reported together with the percentage frequency of the total concerns per section and subsection of the CTD, which was calculated as follows:

Percentage frequency of concerns identified $=($ Absolute frequency of specific concern adopted $L$

Total number of concerns adopted per sub/section of CTD)_× 100 .

Data are expressed as nominal values. All charts and analysis were carried out with Microsoft Office Excel 2003 (Microsoft Corporation, USA).

\section{RESULTS}

\section{Descriptive Statistics on CHMP-Adopted Day 120 List of Questions}

The CHMP identified and adopted a total of 1074 deficiencies during evaluation of the quality modules of the CTD dossiers for 22 biosimilar MAAs at day 120 of the centralised procedure. These consisted of 32 'major objections', with 15 'major objections' for drug substance and 17 for drug product, and 1042 'other concerns', with 547 'other concerns' for drug substance and 495 for drug product.
The results show that there is an approximately 1:1 distribution ratio of deficiencies between drug substance and drug product. The mean number of deficiencies for a biosimilar application was $1.45(0-12)$ for 'major objections' and 47 (44-131) for 'other concerns'.

\section{Analysis of 'Major Objections' Adopted by CHMP at Day 120}

Of the 15 'major objections' on drug substance for biosimilar applications adopted by the CHMP, the most prevalent deficiencies were related to biocomparability $(n=4)$, analytical method validation $(n=2)$, reference standards or materials $(n=2)$, stability $(n=2)$, processes $(n=1)$, specifications $(n=1)$, drug substance comparability $(n=1)$, sourcing of reference product $(n=1)$, and characterisation $(n=1)$. 
Table I. Biosimilars with Finalised Assessment Procedure in the EU by End of October 2015 (8)

\begin{tabular}{|c|c|c|}
\hline Trade name (active substance) & Marketing authorisation holder & Status \\
\hline Omnitrope ${ }^{\mathrm{TM}}$ (somatropin) & Sandoz & Authorised \\
\hline Valtropin ${ }^{\mathrm{TM}}$ (somatropin) & Biopartners & Authorised-withdrawn ${ }^{*}$ \\
\hline Alpheon ${ }^{\mathrm{TM}}$ (interferon alfa) & Biopartners & Negative outcome \\
\hline Binocrit $^{\mathrm{TM}}$ (epoetin alfa) & Sandoz & Authorised \\
\hline Epoetin alfa Hexal ${ }^{\mathrm{TM}}$ (epoetin alfa) & Hexal & Authorised \\
\hline Abseamed $^{\mathrm{TM}}$ (epoetin alfa) & Medice & Authorised \\
\hline Silapo ${ }^{\mathrm{TM}}$ (epoetin zeta) & Stada & Authorised \\
\hline Retacrit $^{\mathrm{TM}}$ (epoetin zeta) & Hospira & Authorised \\
\hline FilgrastimRatiopharm ${ }^{\mathrm{TM}}$ (filgrastim) & Ratiopharm & Authorised—withdrawn ${ }^{*}$ \\
\hline Ratiograstim ${ }^{\mathrm{TM}}$ (filgrastim) & Ratiopharm & Authorised \\
\hline Biograstim $^{\mathrm{TM}}$ (filgrastim) & CT Arzneimittel - AbZ-Pharma & Authorised-withdrawn ${ }^{*}$ \\
\hline Tevagrastim $^{\mathrm{TM}}$ (filgrastim) & Teva & Authorised \\
\hline Filgrastim Hexal $^{\mathrm{TM}}$ (filgrastim) & Hexal & Authorised \\
\hline Zarzio $^{\mathrm{TM}}$ (filgrastim) & Sandoz & Authorised \\
\hline Nivestim $^{\mathrm{TM}}$ (filgrastim) & Hospira & Authorised \\
\hline Remsima $^{\mathrm{TM}}$ (infliximab) & Celltrion & Authorised \\
\hline Inflectra $^{\mathrm{TM}}$ (infliximab) & Hospira & Authorised \\
\hline Ovaleap $^{\mathrm{TM}}$ (follitropin alfa) & Teva & Authorised \\
\hline Grastofil $^{\mathrm{TM}}$ (filgrastim) & Apotex & Authorised \\
\hline Bemfola $^{\mathrm{TM}}$ (follitropin alfa) & Finox Biotech AG & Authorised \\
\hline Abasria $^{\mathrm{TM}}$ (insulin glargine) & Eli Lilly & Authorised \\
\hline Accofil $^{\mathrm{TM}}$ (filgrastim) & Accord Healthcare & Authorised \\
\hline
\end{tabular}

* Products whose status is labelled as 'Authorised—withdrawn' were withdrawn by the applicant for commercial reasons

With regard to drug product, 17 'major objections' were raised on process validation $(n=3)$, reference standards or materials $(n=3)$, analytical method validation $(n=2)$, comparability $(n=2)$, inadequate evidence for biosimilarity $(n=2)$, stability $(n=2)$, specifications $(n=1)$, devices $(n=1)$, and GMP compliance $(n=1)$. Tables IV and V list the 'major' objections adopted by the CHMP at day 120 for these biosimilar applications.

\section{Analysis of 'Other Concerns' Adopted by the CHMP at Day 120}

Figure 1 presents the percentage frequency of 'major objections' together with 'other concerns' adopted by the
CHMP at day 120 for drug substance (Section 3.2.S) and drug product (Section 3.2.P).

The frequency of concerns raised by the CHMP results in the following percentage frequency sequence for drug substance (Fig. 2): 36\% for manufacture issues (Section 3.2.S.2), 24\% related to characterisation (Section 3.2.S.3), $23 \%$ on control of drug substance (Section 3.2.S.4), 7\% on each of reference standards or materials (Section 3.2.S.5) and stability (Section 3.2.S.7) and 1\% on container closure system (Section 3.2.S.6).

The results show that that the majority of 'other concerns' raised for drug substance relate to issues concerning manufacture, characterisation and control of drug substance, since together they amount to over $80 \%$ of the total raised 'other concerns' for drug substance.

Table II. CTD-Based Sections and Subsections for Classification of Observations for Drug Substance

\begin{tabular}{|c|c|}
\hline $\begin{array}{l}\text { C T D } \\
\text { section }\end{array}$ & Section title and subsections content \\
\hline 2.S.1 & General Information: 3.2.S.1 Nomenclature, 3.2.S.2 Structure, 3.2.S.3 General Properties \\
\hline 3.2.S.2.1 & $\begin{array}{l}\text { Manufacture: 3.2.S.2.1 Manufacturer(s), 3.2.S.2.2 Description of Manufacturing Process and Process Controls, 3.2.S.2.3 Control of } \\
\text { Materials, 3.2.S.2.4 Control of Critical Steps and Intermediates, 3.2.S.2.5 Process Validation and/or Evaluation, 3.2.S.2.6 } \\
\text { Manufacturing Process Development }\end{array}$ \\
\hline 3.2.S.3 & Characterisation: 3.2.S.3.1 Elucidation of Structure and Other Characteristics, 3.2.S.3.2 Impurities \\
\hline 3.2.S.4 & $\begin{array}{l}\text { Control of Drug Substance: 3.2.S.4.1 Specifications, 3.2.S.4.2 Analytical Procedures, 3.2.S.4.3 Validation of Analytical Procedures, } \\
\text { 3.2.S.4.4 Batch Analysis, 3.2.S.4.5 Justification of Specifications }\end{array}$ \\
\hline 3.2.S.5 & Reference Standards or Materials \\
\hline 3.2.S.6 & Container Closure System \\
\hline 3.2.S.7 & $\begin{array}{l}\text { Stability: 3.2.S.7.1 Stability Summary and Conclusions, 3.2.S.7.2 Post-approval Stability Protocol and Stability Commitment, } \\
\text { 3.2.S.7.3 Stability Data }\end{array}$ \\
\hline
\end{tabular}


Table III. CTD-Based Sections and Subsections for Classification of Observations for Drug Product

\begin{tabular}{ll}
\hline $\begin{array}{l}\text { C T D } \\
\text { sections }\end{array}$ & Section title and subsections content \\
\hline 3.2.P.1 & $\begin{array}{l}\text { Description and Composition } \\
\text { Pharmaceutical Development: 3.2.P.1 Components of the Drug Product, 3.2.P.2 Manufacturing Process Development, 3.2.P.3 } \\
\text { 3.2.P.2 } \\
\text { Container Closure System, 3.2.P.4 Microbiological Attributes, 3.2.P.5 Compatibility }\end{array}$ \\
3.2.P.3 & $\begin{array}{l}\text { Manufacture: 3.2.P.3.1 Manufacturer(s), 3.2.P.3.2 Batch Formula, 3.2.P.3 Description of Manufacturing Process and Process } \\
\text { Controls, 3.2.P.3.4 Controls of Critical Steps \& Intermediates, 3.2.P.3.5 Process Validation and/or Evaluation }\end{array}$ \\
3.2.P.4 & $\begin{array}{l}\text { Control of Excipients: 3.2.P.4.1 Specifications, 3.2.P.4.2 Analytical Procedures, 3.2.P.4.3 Validation of Analytical Procedures, } \\
\text { 3.2.P.4.4 Justification of Specifications, 3.2.P.4.5 Excipients of Human or Animal Origin, 3.2.P.4.6 Novel Excipients }\end{array}$ \\
3.2.P.5 & $\begin{array}{l}\text { Control of Drug Product: 3.2.P.5.1 Specifications, 3.2.P.5.2 Analytical Procedures, 3.2.P.5.3 Validation of Analytical Procedures, } \\
\text { 3.2.P.5.5 Batch Analyses, 3.2.P.5.5 Characterisation of Impurities, 3.2.P.5.6 Justification of Specification }\end{array}$ \\
3.2.P.7 & $\begin{array}{l}\text { Reference Standards or Materials } \\
\text { Container Closure System }\end{array}$ \\
& $\begin{array}{l}\text { Stability: 3.2.P.8.1 Stability Summary and Conclusion, 3.2.P.8.2 Post-approval Stability Protocol \& Stability Commitment, 3.2.P.8.3 } \\
\text { Stability Data }\end{array}$ \\
\hline
\end{tabular}

Analysis of the percentage frequencies for drug product 'other concerns' results in the following sequence (Fig. 3): $29 \%$ for manufacture issues (Section 3.2.P.3), 26\% related to control of drug product (Section 3.2.P.5), 18\% on stability (Section 3.2.P.8), 7\% each on pharmaceutical development (Section 3.2.P.2) and container closure system (Section 3.2.P.7), 6\% for control of excipients (Section 3.2.P.4), $4 \%$ on reference standards or materials (Section 3.2.P.6) and $2 \%$ related to general information (Section 3.2.P.1).

The results show that the majority of 'other concerns' raised for drug product were related to issues concerning the manufacture, control of drug product and stability as together they amount to over $70 \%$ of the total raised 'other concerns' for drug product.

Analysis of these concerns show that some similarities exist in the percentage frequencies identified between drug substance and drug product. The frequently raised concerns were on manufacture issues (36 and 29\% for Sections 3.2.S.2 and 3.2.P.3, respectively) and control of drug substance and drug product (23 and 26\% for Sections 3.2.S.4 and 3.2.P.5, respectively), followed by concerns on characterisation for drug substance (Section 3.2.S.3) and 18\% on stability for drug product (Section 3.2.P.8).

Further analysis (Table VI) reveals that for control of drug substance (Section 3.2.S.4), 94\% of concerns were due to (1) inadequate validation of the analytical procedures $(33 \%)$, (2) issues with regard to the applied analytical procedures $(22 \%)$, (3) inadequacy or lack of certain specifications $(21 \%)$ and (4) lack of justification for the adopted specifications $(18 \%)$.

For the manufacture of drug substance (Section 3.2.S.2), $98 \%$ of the deficiencies concerned the following: (1) lack of details regarding the description of manufacturing process and process controls $(29 \%)$; (2) inadequate process validation and evaluation (28\%); (3) inadequate control of materials used in the manufacturing processes $(16 \%)$ and of critical steps and intermediates (15\%); and (4) lack of details, data or description of the manufacturing process development (10\%).

For characterisation (Section 3.2.S.3), 76\% of the observations made concerned inadequate elucidation of the structure and characteristics of the drug substance, with the remaining $24 \%$ being associated with the lack of data or discussion on whether starting materials contribute to impurities of the drug substance and lack of justification for the accepted levels of impurities.

The remaining concerns adopted by the CHMP related to reference standards and materials (3.2.S.5) 7\%, container closure system (3.2.S.6) $1 \%$ and stability (Section 3.2.S.7) 7\%. The majority (62\%) of concerns associated with stability were due to lack or inadequate data supporting the proposed shelf lives, while 22 and $16 \%$ were related to inadequate stability summary and conclusion/discussion, and deficient postapproval stability protocols and stability commitments. respectively.

With respect to drug product, the majority of concerns were associated with manufacture (29\%) and control of drug product $(26 \%)$ and concerning stability conclusions $(18 \%)$.

Further analysis (Table VII) revealed that for control of drug product (Section 3.2.P.5), 98\% of concerns were due to (1) lack of adequate justification for specifications (24\%); (2) issues with proposed specifications such as wide adopted limits, missing specification tests and inadequately supported specification values $(23 \%)$; (3) improper validation of analytical procedures $(22 \%)$; and (4) missing, inconsistent or insufficient data for batch analysis (16\%) and missing data/ information or insufficiently robust analytical procedures, as well as unjustified multiple use of different analytical procedures for the same analyte $(13 \%)$.

For the manufacture of drug product (Section 3.2.P.3), $89 \%$ of the concerns were related to (1) lack of documentation with regard to the description of manufacturing process and process controls, for example missing details on holding times for starting materials, intermediates and bulk $(28 \%)$; 
Table IV. Major Objections for Drug Substance

Number of major objections Category of deficiency

for drug substance

4
Major objections concerned inadequate evidence of biocomparability between product substance and reference product substance due to lack of supporting data.

Issues identified included:

- Insufficient data for differences in receptor binding (due to secondary mechanisms of action) not explained with respect to observed slight differences in efficacy and safety and hence their clinical impact and impact on assay_-shortcomings and apoptosis testing.

- High levels of oligosaccharide residues influencing the immunogenic potential as compared to the reference product.

- Unusual glycan structures, oligosaccharide residues, have been detected at relatively high levels which structures cannot be considered to reflect normal heterogeneity of $\mathrm{N}$-glycan structures and consequently the impact of this structure (quantitative) on safety and efficacy is unknown.

Major objections regarded insufficient data/evidence or incomplete exercise for analytical validation. Issues raised were concerned with lack or inadequate presentation of the validation of analytical procedures due to:

- Lack of adequate control of the drug substance on a routine basis, the relevant guidelines being only partly considered. Complete revision of the validation of analytical procedures was required. The deficient validation of analytical procedures jeopardises the validity of the batch results, the specifications and the stability data.

- The validation reports for analytical procedures for routine quality control were considered inadequate as they were not in line with ICH Q2A and Q2B (10).

Major objections regarding reference standards and materials used.

Issues identified were:

- Missing information on the exact potency and content of the reference batches used;

- Lack of defined or characterised reference materials;

- Lack of transparency and documentation of reference materials used and of the relative quality:

- Lack of potency and content of batch used as reference product. In the absence of an internationally recognised reference preparation, the content of primary in-house references must be established by absolute methods.

- The establishment of the standards and reference materials was not clear with issues of calibration and qualification was questionable. Information on how in-house reference material was established and qualified was missing.

Major objections regarding stability studies carried out.

Issues raised were concerned with:

- Insufficient or inadequate stability data to support the proposed shelf life. There were no real-time and realcondition stability results using the proposed container closure system. Results from ongoing studies, as well as real-time stability data for the entire proposed storage were missing;

- Insufficient stability data on batches tested using validated assays and produced through validated processes; - Lack of temperature monitoring during transport across temperature zones.

Major objection was raised on the process control strategy used which was not clear and not enough data was submitted to support the design space approach used. Critical quality attributes were not identified, based on product characterisation and clinical evidence, to quantify the design space.

Major objection was raised with regard to the specification of drug substance not being sufficiently justified and being impaired by insufficient validation of the analytical method used. The specifications did not take into consideration related substances and the actual batch results in conjunction with the respective precisely validated methods. A revised drug substance specification was required after final and complete characterisation of the drug substance based upon adequately validated assays.

Major objection was raised with regard to two different but similar manufacturing processes whose comparability was not adequately shown. Clinical trials and biosimilarity study exercises used one manufacturing process while the commercial manufacturing will use another process which is similar but with several process adjustments to optimise manufacturing. Comparability between these processes was not considered to have been demonstrated due to the lack of information provided regarding the nature of some of the process changes implemented and the limited degree of testing applied.

Major objection raised concerning reference product used which was not authorised in the EU and the same reference product was not used throughout the comparability program for quality, safety and efficacy studies.

Major objection was raised regarding the characterisation of the drug substance, which was considered inadequate since the techniques employed do not ensure that the methodologies employed were of a sufficiently discriminating nature. Also characterisation of impurities was inadequate. Each chosen technique was not justified and appropriately qualified. 


\section{Number of major objections Category of deficiency}

for drug product

3

3
Major objections concerned insufficient data and evidence for process validation

Issues identified included:

- The aseptic manufacturing process for the drug product was not considered validated at full proposed production scale size for the final product strengths and not for all the strengths. Respective validation data considering the extremes of formulation strength, bulk solution size and number of syringes were not presented.

- Formal validation of the manufacturing process was not performed. This was required once analytical procedures have been rigorously validated, and the facilities and processes employed re-evaluated and deficiencies addressed.

Major objections regarded issues with reference standards or reference materials used. Issues raised were concerned with:

- No information was submitted on the exact potency and content of the reference batches used;

- Ill-defined and characterised reference materials;

- The primary reference material and working reference material for the active substance were not clearly defined and characterised.

Major objections regarded insufficient data and evidence or incomplete method and exercise for the validation of analytical methods used.

Raised issues were concerned with:

- The presentation of the validation of analytical procedures for identity, purity and assay were completely inadequate and did not provide evidence for the adequate control of the drug product on a routine basis;

- Method validation was not completed at the time of submission;

- The applicant did not determine that each test had been appropriately validated for the intended purpose;

- Properly constructed and completed validation reports for all tests are lacking;

- The deficient validation of analytical procedures jeopardises the validity of the batch results, the specifications and the stability data.

Major objections regarded insufficient data for drug product comparability, namely insufficient structural characterisation data or comparisons done on drug product.

Direct comparability exercise between the drug product obtained with the definitive process and the reference product was not performed. Therefore, state-of-the-art direct proof that the definitive production process results in a drug product whose structural aspects are identical to a drug product licenced in the EU was not presented. Major objections regarded insufficient or inadequate stability data to support the proposed shelf life of finished drug product.

Main issues raised concerned:

- The stability programme should be revised after proper validation of the analytical methods and any consequent revision of the finished product specification. The parameters tested in the stability should adequately reflect stability-indicating parameters, especially as regards purity testing;

- Data was not provided for three production batches, manufactured under a satisfactorily validated process and to specifications demonstrated to be robust with respect to limits and the analytical procedures employed.

Major objections concerned with biosimilarity between product and reference product were not considered as established due to lack of supporting data.

Issues raised in this context included:

- Insufficient data was used, namely low number of EU batches analysed for several tests, not in line with the CHMP biosimilar guidance, which states that several different batches of the reference product should be used to provide a robust analysis and to generate a representative quality profile;

- Differences in receptor binding, due to secondary mechanisms of action, were not explained with respect to observed slight differences in efficacy and safety; hence, their assay and clinical impact were not demonstrated. Major objection was raised with regard to the specifications of the finished drug product as not being justified. The main issues raised for this lack of justification were inadequate:

- Inclusion of related products in finished drug product;

- Drug substance characterisation; and

- Validation of analytical methods used.

Major objection was raised with regard to the use, without any justification whatsoever, of different primary packaging material/devices in production to those used in clinical trials, which also had a higher impurity profile. Major objection was raised for the lack of GMP compliance evidence throughout the dossier both for the clinical batches used in the clinical trials and for batches used in all validation studies.
(2) inadequate validation and evaluation for manufacturing processes, including those for critical analytical tests used during manufacturing including production scale manufacturing (47\%); and (3) unjustified or missing critical data such as testing frequency for adopted controls of critical steps and intermediates $(14 \%)$.

The other $11 \%$ of the observed concerns for the manufacture of drug product (Section 3.2.P.3) were related 


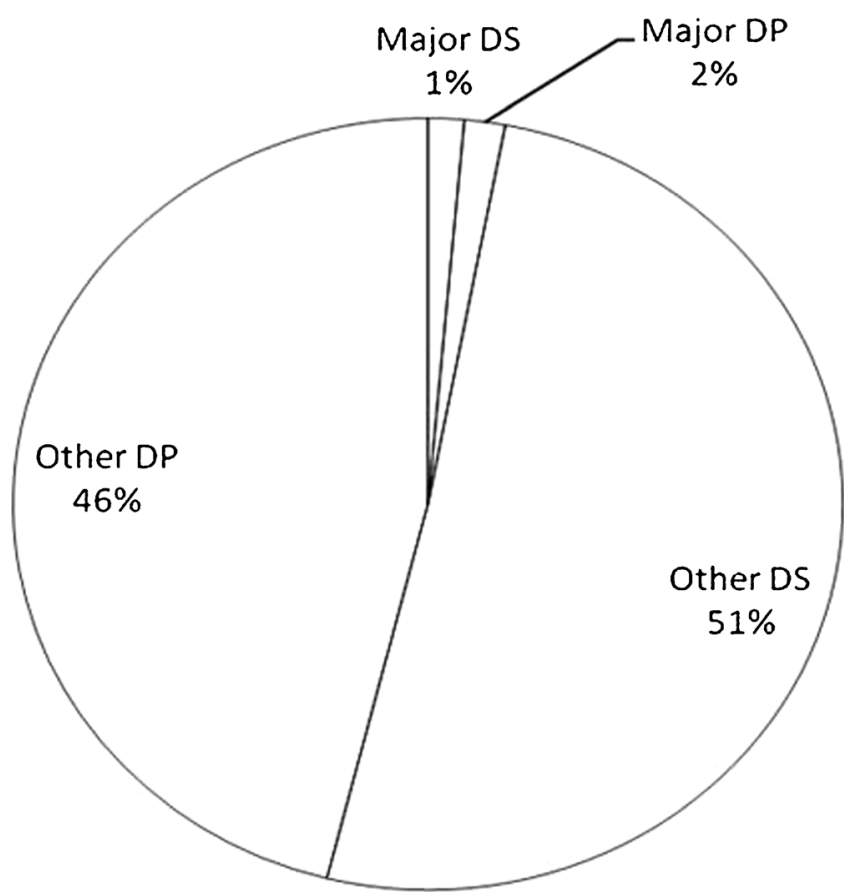

Fig. 1. Percentage frequency of quality issues adopted as major objections and other concerns for drug substance (DS) and drug product (DP) at day 120 by CHMP for marketing authorisation applications of biosimilars concluded up till end of October 2015

to missing information and GMP certificates for manufacturers $(2 \%)$; incomplete, inconclusive or unclear manufacturing batch formulae (3.5\%); and unjustified or improperly validated transport and storage conditions including missing related reports or data $(5.5 \%)$.

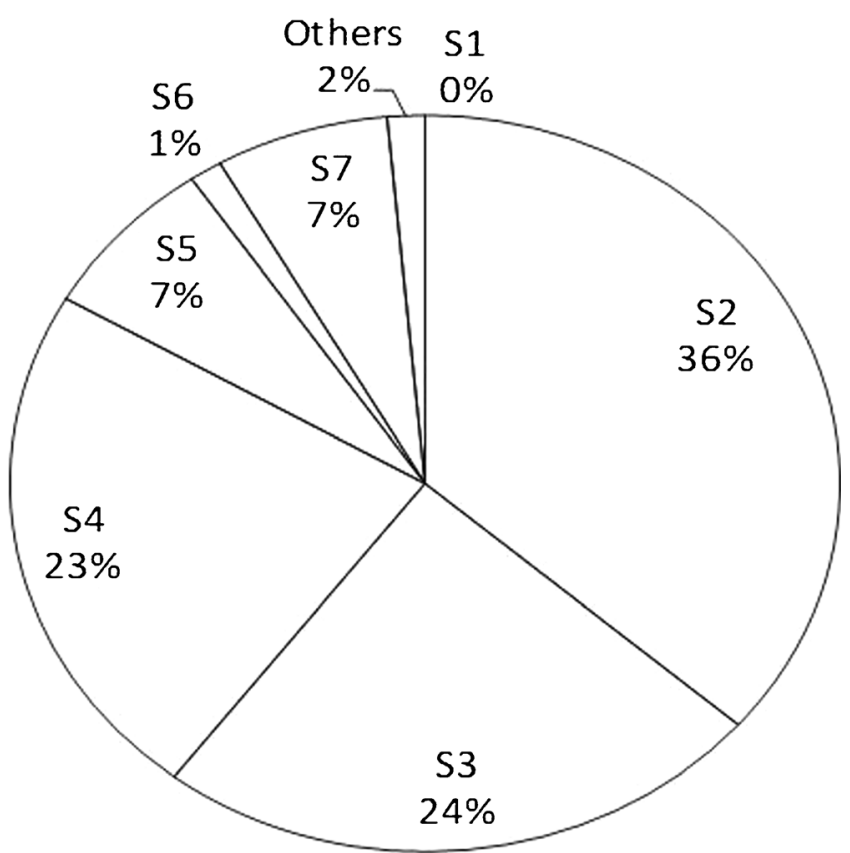

Fig. 2. Percentage frequency of quality 'other concerns' adopted on drug substance $(n=547)$ at day 120 by CHMP for marketing authorisation application of biosimilars concluded up till end of October 2015. Labels denote the different sections of the CTD quality

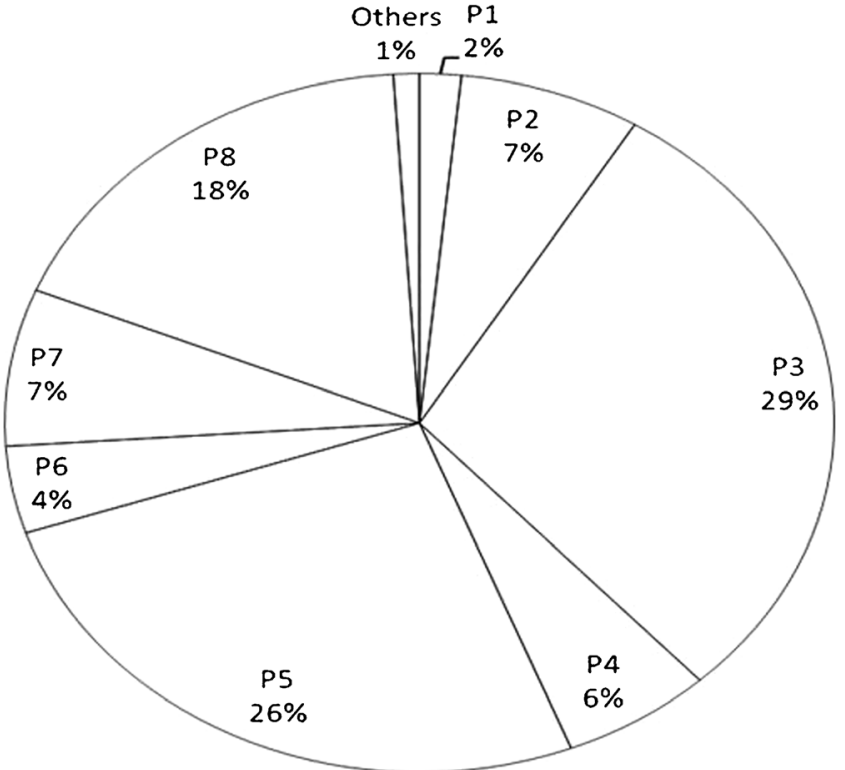

Fig. 3. Percentage frequency of quality 'other concerns' adopted on drug product $(n=495)$ at day 120 by CHMP for marketing authorisation application of biosimilars concluded up to October 2015. Labels denote the different sections of the CTD quality

\section{DISCUSSION}

In this study, all deficiencies ('major objections' and 'other concerns') raised by the CHMP at day 120 during the scientific evaluation of dossiers submitted to EMA through the centralised procedure were reviewed. To the authors' knowledge, this is the first study reviewing biosimilar quality deficiencies adopted by regulators when assessing the risk/benefit of medicinal products during the MAA application stage. Described deficiencies at the level of CTD offer a comprehensive report and analysis of the most prevalent issues encountered in the registration process of biosimilars within the EU.

The obtained results show that there are common deficiencies observed by the CHMP during the scientific evaluation of the dossiers for biosimilar MAAs. The deficiencies for drug substance parallel well the most prevalent deficiencies observed for drug product. The similarity in observed deficiencies for both drug substance and drug product components of biosimilar products should not be taken as a surprise when considering that one incorporates the other and hence characteristics of the substance will inevitably affect those of the product and both have to follow the same guidelines (14).

When comparing these commonly observed deficiencies for biosimilar products with those observed by the same scientific body, i.e. by the CHMP for chemical entities as reported by Borg et al. (15), one would observe a different emphasis. In their analysis of all day $120 \mathrm{CHMP}$-adopted list of questions for a total of 52 MAAs of chemical medicinal products during the two-year period (2007-2008), Borg et al. (15) reported that the most prevalent observations (both 'major objections' and 'other concerns') for drug substance were related to the control of drug substance and the specification, possible qualification and profiling of impurities, including genotoxic impurities, and stability of drug substance, whereas for drug product, the most prevalent 
Table VI. 'Other Concerns' for Drug Substance

Category of deficiency

Absolute $\%$ frequency of frequency of concern/specific concern (f) CTD module section

Module 3.2.S.1-General information

There were no concerns raised under this module section.

Module 3.2.S.2-Manufacture

Section 3.2.S.2.1-Observations related to GMP aspects and details of manufacturers:

0

- GMP certificates were missing for manufacturing, testing, batch release and/or importation sites with no information on last inspection date.

Section 3.2.S.2.2-Observations related to description of manufacturing process and process controls included: 57

- There was missing information and/or description for: cell lines used and their conformity with ICH; WCBs 16 and their genetic stability; plasmid integrity; expression vectors and cloning steps; unexplained mismatches in gene sequence of vector used and annotated specified human c-DNA sequence; at what level/stage is the bacteriophage test carried out; inaccurate description of cell disruption unit operation; incomplete or missing data in genetic stability studies; and unclear origin or batches from single vial of the WCB.

- Incongruent results were presented throughout the dossier for biological activity of manufacturing runs.

- IPC issues included: unclarity whether reprocessing is allowed; reference was lacking for IPC tests; rationale 4 missing or unclear for identifying critical steps; missing reject limits for IPC critical parameters and which batches were used for statistical determination of IPC limits; IPC limits not based on experience gained through development phase and on limits challenged in validation; some IPC tests had no upper and lower limits; and bio-burden control limits not specified for some process stages.

- Validation issues included: manufacturing steps not in line with validated production parameters; no specifications for validated shipping cool packs; lack of valid data showing correlation of cell line expansion between the sampling flask and production inoculum flask; missing validation studies for specific steps, e.g. purification of dialysis tubes used.

- Strategy for EU and non-EU processes segregation was not given when using same equipment;

- Criteria of how fractions are determined in the (specified) chromatographic step during pooling is missing.

- The action taken should the biomass wet weight not achieve the specified action limit should be confirmed.

- It should be confirmed how the active concentration range from the ultrafiltration operation feeds into the $\mathrm{pH}$ precipitation steps.

- It was unclear why there was a relatively large difference between the oligosaccharide results for the in vitro cell age limit cells and EPC.

- It was not clear what the passage number was for the 'in vitro cell age at harvest of routine production' based on data from batches at production scale to date.

- It was unclear whether batches of media used for validation were of the same formulation or not.

- Manufacturing steps issues included: discrepancies in descriptions of DS purification process and storage specifications; missing information on holding steps and on biomass containers storage and their cleaning; discrepancies in buffer concentrations used and agitation speeds at different fermentation steps; unjustified extension of the seeding train; missing description of how the operating criterion for solubilisation was reached; $80 \%$ only viability at the upstream process level was not justified; missing description and time for process of batches from initiation of harvest to end; and lack of details of antifoaming used.

Section 3.2.S.2.3-Observations related to control of materials included:

- The introduction of a polymerase chain reaction (PCR) test for minute virus mice (MVM) in the harvest should be considered, unless otherwise justified.

- Issues with test methods included: questionable accuracy of test methods used; unspecified bio-burden and 5 endotoxin limits for some manufacturing steps; specification limits for production IPCs not based on data from at least five batches; few electropherograms were submitted to show genetic stability; and there were unexplained chromatographic pictures in the dossier.

- Data should be provided clarifying whether rearranged expression vectors were retrieved in the characterisation of the MCB or whether the apparent difference in the estimated fraction of antibioticresistant cells and the cells harbouring the (specified) DNA sequence resides from example variability of the methods used for analyses.

- There was missing data in relation to: proposed protocol for control of stored cell banks' stability; data 3 confirming heterothallic state and mating type of producer cell strain.

- Issues with control of the MCB and WCB included: lack of description for cell age; inconsistent 10 characterisation of WCB; unjustified specification limits for MCB and WCB recombinant construct; unjustified acceptance criteria or tests for cell viability and productivity for in vitro cell age and for WCB stability.

- The osmolality in the (specified) medium was close to the lower limit, especially after the change in pH adjustment. Therefore, the potential impact of this observation should have been discussed.

- Observations related to the used raw materials included: the list of suppliers for non-compendial materials 5 that are tested according to in-house specifications was not provided and whose specifications should be 
Table VI. (continued)

Category of deficiency

Absolute $\%$ frequency of frequency of concern/specific concern (f) CTD module section

justified; controls for compendial materials were not confirmed; there were unclarified discrepancies between manufacturers and receipt testing conductivity specifications for some buffers.

- The following two parameters to monitor the storage stability of the cell banks required justification or suitable adaptation: (a) the acceptance criterion cell count: viable cell number and (b) the restriction enzyme analysis digestion.

- The last batch shown in a series of consecutive batches had a drastically reduced copy number. So the copy number of the next batch should have been revealed and if it approached the limit, the rejection criteria should be adjusted to reflect the present copy number data.

- Stability of medium used not shown and quality/grade of any hormones used in cell culture medium not 2 specified.

- Confirmation required that no materials of human and animal origin were used for the recombinant aminopeptidase production.

Section 3.2.S.2.4-Observations related to controls of critical steps and intermediates included:

- The control strategy was not fully in line with principles and definitions described in ICH Q8-R2 (10).

- Glycosylation step control was not demonstrated for consistency.

- No upper time limits were given for stirring or for other in-process controls to determine that adequate solubilisation has occurred.

- Cultivation or sampling time after induction for produced batches was not as per defined critical process 2 parameter.

- There was missing or lack of data: supporting acceptable ranges listed in the parameter evaluation reports for proposed commercial processes; for productivity at harvest stage; asserting that nutrient levels are not limiting some stages of growth and fermentation; assessing appropriateness of acceptance criteria for some critical parameters such as standard deviation of specifications and bio-burden specifications; missing for some control methods for stability testing of intermediates.

- Issues with key performance parameters (KPPs) included: the relationship of the expected range for KPPs 2 with the classification of parameter ranges defined in the parameter evaluation report was not clarified; actions of the active substance manufacturer should be confirmed in cases when designated KPPs do not meet the expected range indicated.

- There were inconsistencies in the documented control strategy and parameter classification for production fermentation agitation rate, airflow and oxygen mass transport.

- Observations related to control of test methods included: unjustified operating ranges on chromatography bed heights; only brief descriptions of analytical procedures were presented; in-process testing throughout all production is done for information only with no acceptance or rejection criteria set; unjustified absence of minimum/maximum loading limits on columns; unjustified limits and choice of analytical test procedures.

- Compatibility of the specification for the isolated inclusion body intermediate with the proposed manufacturing process should be discussed.

- Information on cut-off limits in use during collection of chromatographic fraction, as well as on the criteria for pooling fractions, was missing.

- It was not specified under which circumstances reprocessing of the filtration steps can be allowed, and the number of times it can be allowed at the final filtration and filling stage.

- A number of in-process controls should be tightened to reflect the results obtained, and the controls on the 2 duration of cultivation/fermentation should not be defined as operation controls, in view of the substantial plasmid loss at the main fermentation.

- For all columns, no parameters such as column diameters and linear flow rates were given.

- There was no confirmation that no freeze/thaw cycles of frozen capture pools are intended.

- There were no criteria when medium is considered exhausted and no acceptance criteria on harvest viability and on degree of purity at purification steps which should be reviewed together with action limits and operational parameters after more experience is gained.

Section 3.2.S.2.5-Observations related to process validation and/or evaluation included:

- Inconsistencies were shown in relation to: validation of HCP and endotoxin removal; HPLC results for virus 8 inactivation step with some batches outside expected range; maximum hold time for critical processes; drug substance harvests; DS-related impurity levels determined by HPLC and those presented in dossier tables.

- A maximum number of allowable re-uses for chromatographic columns used in purification should be defined, based on appropriate validation studies and for columns claimed to contribute to virus safety, and virus removal capacity should be validated for aged column material, which is representative for the specified column lifetime.

- The longest process time in production runs is unjustified.

- There were missing data in relation to or showing: re-evaluation of process parameters and IPC for 22 fermentation and tangential flow filtration membranes; no process-related impurities from chromatography columns are carried over to the bulk DS; on bio-burden control throughout purification process spiking studies 
Table VI. (continued)

Category of deficiency

for removal validation of process-related impurities due to low levels which are confirmed by the developed analytical methods; proposed storage time of intermediates and/or process validation; impact of extreme conditions on DS during viral inactivation; cleaning process efficiency of chromatography columns; to demonstrate consistency in the manufacture of validation batches; chromatographic elution profiles for the validation batches; validation for re-use of gel filtration column; maximum culture time; 6 months stability for intermediates, endotoxin and bio-burden; for small-scale evaluation studies of the process, validation is lacking; on the level of glycosylation at fermentation end; removal of specific process-related impurities; chromatography matrices leachables testing.

- Clarification was required on the reason for repeating a step in two of the validation runs.

- The formation of correct disulphide bridge pairing was not validated using several batches and appropriate analytical procedure.

- There were unqualified or unvalidated items: reusable materials such as chromatographic columns and resin; 7 cleaning for re-used equipment; repeat of nano-filtration step in case of failed filter integrity test; transport of DS to DP manufacturer.

- Process parameters for the purification process were defined but their suitability and appropriateness was not demonstrated.

- Only four batches were used for the determination of operational parameters, acceptance criteria and specifications for the commercial process. These should be reassessed for the USP and DSP using a larger number of full-scale batches, since some limits appeared rather wide.

- Downscaled models for column sanitisation validation were not acceptable.

- Acceptance and rejection criteria for the allowed column runs were not set up or specified based on 3 validation data.

- Analytical results and column-elution profiles of the full-scale process validation studies were not provided.

- There is no guarantee included in the fermentation and purification in-process controls, suitable tests and acceptance limits that the operating parameters and quality attributes remain within the validated ranges.

- Doubling time should have been re-established as an acceptance criterion for cell expansion and not replaced by the PDL parameter.

- Studies for the extension of the column lifetime have to be submitted for approval via the variation procedure.

- Commitment was required for batch analysis of DS produced with intermediates stored at the maximal holding time.

- There were unjustified items: viability acceptance criteria for cell expansion and harvest productivity; action 4 limit for whole production processing; non-evaluation of some critical operating parameters.

Section 3.2.S.2.6-Observations related to the manufacturing process development included:

- There were unjustified items: upper limits for dissolved oxygen and glucose during seeding and culture 8 production; predefined limits for process parameters and some IPCs; high production yields of some runs compared to others; inclusion of some components in buffers used for dissolution of inclusion bodies; HCP protein amounts in batches manufactured initially and later in development; different fermentation runs used in the production of pre-clinical trial material and market final product; differences in yields of validated batches; lifetime of ultra and difiltration membranes.

- The following were unspecified or unclarified: water grade used in some processes; storage site locations; 4 stability of antifoam under sterilisation conditions; whether plasmids express other proteins.

- An estimate of the generation number of the EPC and the PPC used for the genetic stability studies was not given.

- There was missing information in relation to: back-up storage site for MCB and WCB; IEX to allow a more 2 dilute process stream.

- Column loads studied were lower than the defined maximum and the maximum column runs were not 3 supported by data.

- Discussion was required whether the variations in $\mathrm{HCP}$ depletion can be correlated to the implementation of two different HCP standards that differ in the number of purification steps performed for production.

- The difference in control of cation exchange chromatography column loading between the defined processes was not discussed.

Module 3.2.S.3 - Characterisation

Section 3.2.S.3.1 - Observations related to the elucidation of structure and other characteristics included:

- N-terminal sequencing has been performed for only few cycles on intact samples and with limited peptide mapping.

- Not all peaks that have been identified were shown on chromatograms for peptide mapping.

- Comparability was not considered as demonstrated since potency data comparing batches with reference 7 product was unclear with respect to for example the assay test method used. 7
Absolute \% frequency of frequency of concern/specific concern (f) CTD module section 
Table VI. (continued)

Category of deficiency

Absolute \% frequency of frequency of concern/specific concern (f) CTD module

- Not all test methods used in reference product characterisation were used in the characterisation of drug section substance under application.

- Amount of aggregates was not determined with an orthogonal method when using SEC-HPLC.

- There were unvalidated methods for: mapping/identity of peptides; analysis of potentially present 7 carbohydrates as suitable for proteins with low carbohydrate content; the isolation procedure of DS; in vitro biological activity of DS batches demonstrated by dose response curves of all batches in comparison with the DS BRP standard.

- There was missing data related to: chromatographic parameters for separation of digested peptides; spectrographs used in characterisation; real-time and accelerated time storage conditions studies for DS and DP; potential leachables from the chromatographic media, together with relevant toxicological and clearance data; the provided comparability and/or characterisation studies; comparative $Z$-values for the proposed glycan distribution specification for some batches of active substances; quantification of sialic acid and its variants.

- The extinction coefficient for DP and DS was not experimentally determined based on amino acid analysis and compared to the theoretical extinction coefficient.

- The identity assignment of the IEC-HPLC fractions required further discussion.

- There were unjustified items: variations in near UV CD spectra for DP; variability for the N-linked glycan analysis in DP batches compared to DS batches; difference between the predicted and observed half-life values of the beta subunit amino acid residue; specification for the isoform set less than the $\mathrm{Ph}$. Eur. value; mass ratio differences observed with the pool using different analytical methods; differences in chromatographic behaviour between peaks; differences observed in some isoforms which raise the question of reliability of the DP sample preparation procedure; sialic acid relative ratios between monosaccharides in the total pool of glycans which were out of the structurally possible ratio range; specific sugar structures which could not be detected of the total glycan pool of DS whereas these structures could be detected in N-glycans of samples where the content of high sugar-type glycans was considerably lower; differences in quantification of sugars between the two test methods used; comparability exercise experiments using different antibodies.

- There was missing explanation or information for: unlabelled graphs; results from an in vitro neutralisation activity for Ds and DP indicating a lower potency in DP; different molar extinction coefficients determined for batches of DS which are used as reference materials; ELISA results showing excess binding; results obtained from the amino acid analysis with regard to the low percentage of specific amino acids in the analysed batches; sodium content in validation batches which varies more than what would be expected; batches used during the DS characterisation testing; differences in chromatographic bands and in truncated forms; clarification of the scoring system used in some provided tables; lack of spectroscopic studies on the three-dimensional structure of DS; why smaller fractions of DS-specific batch that also contained oligosaccharide structures were excluded from analysis; peptide LC-MS data profiles of both the test and reference DP samples showing oxidised peptides and variant form peptide fragments as additional peaks; why no dimers were found in the batches of active substances contradicting the finding that lyophilisates manufactured from the same active substance exhibited a clearly visible band; why no lyophilised product manufactured at one specific site had been included in the rat weight gain assay; how it can be guaranteed that a validated biological activity assay was used to fulfil the minimum $\mathrm{Ph}$. Eur. requirements; peaks occurring in the chromatogram were assigned to certain structures derived from a standard chromatogram; not identifying and verifying elution groups by spiking experiments or by the use of a standard solution; reference being made only to specific linked sugars in the chromatogram of linkage analysis of monosaccharides; binding characteristics of the antibody used in the test demonstrating that the antibody was capable to distinguish between different conformational stages of the DS molecule; differences in deglycosylation of O-glycans between DS batches and reference product samples although the O-glycan structures were characterised as qualitatively similar.

- It is unclear how the protein complex is detected in the assay.

- The results of SEC-HPLC and RP-HPLC were not fully evaluated.

- For determination of purity, related proteins were not differentiated.

- There were two major bands visible in the Western blot test which required further analyses to understand the nature of these molecular species.

- No full reports of the study of the comparative biological activity in the in vivo bioassay were provided.

- Characterisation and comparability studies were not extended with an orthogonal method for (non-covalent) 2 aggregates, and the test method employed did not provide accurate mass values for samples tested.

- Glycosylation issues were observed associated with for example their analysis and data incongruence in 6 quantification calculation of oligosaccharide structures of specific glycosylation sites.

- In the comparability exercise between active substance and reference product batches, the content of total molecular variants was listed only as a sum parameter and did not consider the quantitative level of the individual protein variants. 
Table VI. (continued)

Category of deficiency

- The study design for the in vitro cell proliferation assay revealed that no comparator product had been integrated into the test programme.

- Assurance was required, that all of the $\mathrm{N}$-glycans contained in the prepared sample were found on the chromatogram.

- The validity of proteolytic digestion with respect to length of the resulting peptides was not demonstrated. - In the test method used, the specificity of the binding reaction to the surface-linked anti-drug substanceantibody reaction was only partially demonstrated.

- Demonstration was required showing whether the phosphorylated high sugar-type glycans as well as sulphated complex-type glycans may influence the isoform distribution pattern in the analytical method used. Section 3.2.S.3.2-Observations related to the impurities included:

- Not all process-related impurities besides HCPs and DNA were taken into consideration and risk analysed as 3 well as there was missing discussion on product-related impurities observed during development.

- Removal of impurities was not explicitly addressed in validation studies.

- There were unjustified items: identified impurity peaks; strategy of raising the anti-host cell antiserum; too 6 high antifoam level limits.

- Minor species resolved in analytical methods were not identified.

- There was missing data for: removal of residual DNA throughout the purification process; removal of copper 10 reflecting suitability of applied methods; presence of DS oxidised forms; quantification of unidentified variants; Western blot data demonstrating detected bands in some tests under reducing and non-reducing conditions represent the DS dimmers; potential influence of column lifetime on content of product-related proteins in pooled column elutes.

- A direct comparison of the different forms of the DS was not performed.

- Further clarification was required for: activity of the slow migrating monomer forms of the active substance; 4 impurity profile of enzyme preparation; buffer composition for all DS solutions tested.

- Impurities trending observed during the manufacturing process development were unexplained.

- Presence and clearance of mutants of the active biological molecule in the DS was not discussed.

- The methodology of the analytical tests for detection of charge- and size-based variants in column pools was not described.

- The peptide mass fingerprint of the deglycosylated DS showed additional peaks, and differences in peak intensities which were not discussed.

Module 3.2.S.4-Control of drug substance

Section 3.2.S.4.1-Observations related to the specifications included:

- Specifications were not updated with references to analytical methods SOPs; and specifications' limits and 3 choice of tests were not discussed. Limits were not based on data coming from sufficient number of batches. - Specifications were not based on manufacturing experience and characterisation data, and based on DS 5 batch data, they should be tightened for $\mathrm{pH}$, high molecular weight variants and charge variants; as should be release acceptance criteria for protein concentration.

- Limits did not include control of the appearance of "new" or "unidentified" species in the analyses for purity.

- The level of impurities should be related to the amount of the main product rather than to the intensity of 2 the diluted standard, and the proposed residual DNA limit was not compatible with commonly acceptable limits.

- There was conflicting detail with regard to the method used for endotoxin determination and to some test 2 whether they are used routinely or in stability only.

- There were unjustified items: test limits for total content of dimers, deamidated active and sialic acid; and use 2 of $\mathrm{Ph}$. Eur. method to determine sialic acids when it was shown in the characterisation section that the method of enzymatic cleavage is preferable.

- The DS release criteria for impurity levels were not reflecting production performance and levels observed 10 in clinical batches.

- The raw data of peptide maps was not provided at release and at the latest storage time point available. Section 3.2.S.4.2-Observations related to the analytical procedures included:

- There were unjustified differences or different methods from those in Ph. Eur for: tests for visual inspection 9 of colour and turbidity; total viable counts; test methods used in release control; between test methods used in DS release and in process; biological assay and peptide mapping.

- There were missing data for: detail of the host cell antibody test; detail in description of the analytical 3 procedures; and on sample preparation for potency assay.

- There was an unspecified source for the test kit used to quantify DNA.

- The following required further clarification: the reference standard and method used to normalise the 4 samples' protein content for the bioassay; repeatability and the neutralising and control antibodies used in bioassay validation; which SOPs were used for validated analytical methods; differences between the old and new potency assays used.
Absolute $\quad \%$ frequency of frequency of concern/specific concern (f) CTD module section

0

.


Table VI. (continued)

Category of deficiency

Absolute $\%$ frequency of frequency of concern/specific concern (f) CTD module section

For non-compendial methods, the DS specification was not updated to include reference to relevant SOPs.

- No specifications had been established for the glycosylation.

- The current in-house identification numbers for the test procedures/SOPs were not included.

- The theoretical extinction coefficient used for calculation of the DS concentration was not verified.

- The performance validation of analytical procedures was not in line with recommendations in ICH Q2A and 7 Q2B (11).

Section 3.2.S.4.3-Observations related to the validation of analytical procedures included:

- The following were not validated, or validated properly: only summaries were presented instead of study 23 reports; commercial HCP kits used; HPLC methods used for quantification of product-related impurities; quantification of minor species resolved in methods used for purity control; analytical methods used in stability studies; nature of stress used in stress samples used in validation not confirmed; significant peptide map was not used in the validation of release peptide mapping test; some in-process control method validation did not use a sufficient number of replicates; in vitro bioassay intermediate precision; reference standard was not calibrated against an international standard; linearity of potency assay; DS-specific impurities; linearity for host cell- and vector-derived DNA detection; specificity of gel electrophoresis for impurities; accuracy of HCP analytical method; chromatographic peak homogeneity of active substance; reliable numerical values obtained for purity percentages; detection limit for some specified residuals; test methods used to measure oxidation.

- It was not confirmed that: identity tests are specific for active only and not for other DP analogues; and an 2 extra peak in identity-peptide map was an artefact of the method.

- Further explanation was required for: identified variance between runs; major irregularities or deviations in 7 accuracy validation; control of the refolding steps of the active biological molecules; HCP antibodies used appearing to be auto-reactive; analytical method description of the protein assay and in vitro bioassay potency; details of origin, storage and stability for the HCP standard and capture antibody; calculation formula for some HPLC methods taking into account buffer interference.

- Clarification was required for: LOD and LOQ for HCP analysis methods which deviated from ICH Q2 (11); 3 two protocols with different acceptance criteria for system suitability tests were used in validation for purity control.

- The following were considered as unjustified: acceptance criteria for system suitability tests for analytical 4 methods; appropriateness of analytical test procedure used for in-process testing; specific model protein used for validation of impurities detection.

- Fiducial limits controlling the potency assay were not tightened.

- The statistical model used in the potency assay was not confirmed.

Section 3.2.S.4.4-Observations related to the batch analysis included:

- Consistency in correct disulphide bridge pairing was confirmed based on only one DS batch testing.

- A summary of all batches used in pre-clinical and clinical trials was missing, and statement that all pivotal studies were performed with material from the commercial process was unsubstantiated.

- Batch analysis data based on revised DS specification and validated assays was not provided.

- The batch analysis data tables were incomplete and not sufficiently supported by analytical data coming from 3 selected samples.

- A sufficient number of batches originating from the definitive process were not used for: data on N-terminal 2 variants and batch data on in-process testing.

Section 3.2.S.4.5-Observations related to the justification of specification included:

- Basis of justification not acceptable for: purity limits of DS; acceptable sialic acid levels do not reflect those 5 used in clinical trials; specification limits especially purity limits do not reflect capacity of manufacturing process; sialic acid levels in DS specifications do not reflect those of DS used in clinical trial; and the proposed limits of product-related proteins do not reflect those used in batch results.

- There were unjustified limits for: HCPs; combined use of two methods for ID testing; and the sum of 4 substance-related impurities.

- The following were not considered in specifications: data obtained in stability studies; control for specific 5 receptor-mediated biological activity; capillary zone electrophoresis as routine tests; and tests for $\mathrm{pH}$ and mass determination.

- Release specifications were not based on batches demonstrating manufacturing consistency, safety and efficacy.

- In the analysis for host cell DNA, only primers for amplification of the vector sequences were used.

- The revision of the specification controlling the amounts of higher molecular weight forms of product should be processed as post-approval commitment, and thus, timeframe for fulfilment of the commitment should be defined.

- The following were lacking: a discussion on the specifications, limits, and choice of tests; another quantitative 2 impurity assay to cover all monomeric product-related impurities. 
Table VI. (continued)

Category of deficiency

- Updated batch analysis was not provided on a sufficient number of commercially manufactured batches (of formulated bulk).

- Specifications required confirmation since some contradictory limits were presented.

- The need for routine testing of N-terminal variants was not determined on the basis of sufficient batch data.

- Confirmation was missing that residual AP was routinely determined as an IPC with binding rejection criteria.

Module 3.2.S.5-Reference standards or materials

Observations related to the reference standards or materials included:

- There were issues in relation to the establishment and qualification of the reference standards (RS) such as lack of calibration against international standards, content description and missing information/data or lack of clarity for their characterisation, their preparation, stability, storage container and storage conditions, as well as working standard preparation and shelf life.

- Missing documentation or information for: conformity of in-house standard with DS batch release 9 specifications; origin of dimer and impurities standard used in DS characterisation analytical method validation; used reference standards; CoAs for reference standards; batches of DP used as reference material for analyses in the characterisation of RS; on a secondary (in-house) RS; why batch analysis of some batches was not included in the table of batches.

- Stability indicating impurities was not monitored in the storage of the proposed in-house standard.

- Confirmation was required that: in vitro bioassay potency was qualified against WHO internal standard and 2 its code number is confirmed.

- Clarification required whether original multi-product HCP assay was qualified before use.

- The bioassay for the calibration of the primary RS was not in line with Ph. Eur. monograph.

- Qualification of the primary RS did not include all specified product-related impurities.

- There was no justification for use of data from one specific batch which was not fully characterised in the meta-analysis of analytical characteristics.

Module 3.2.S.6-Container closure system

Observations related to the container closure system (CCS) included:

- There was no information on leachables of the CCS.

- There was no information on the washing procedures, including possible validation reports, of re-used containers and caps.

- The use of containers for DS storage was not supported by stability studies.

- Further investigation of compatibility with immediate container was not provided in view of higher related proteins at high temperatures and active being particularly prone to adsorption.

- There was lack of evidence that the packaging material used for the DS and/or DP was in full compliance with current Ph. Eur.

Module 3.2.S.7-Stability

Section 3.2.S.7.1-Observations related to the stability summary and conclusions included:

\section{9}

(1)

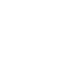

- Retest period not acceptable but a validated expiry was required; shelf-life extension cannot be done on an 2 annual re-analysis.

- Storage conditions were not clear, and storage hold time was not based on more than one batch.

- The batch release and shelf-life specifications for the DS were not in line with ICH Q6B (12).

- It was not confirmed that there were no unidentified species in analyses for purity in progress of stability studies.

- The demonstrated repeated cycles of freezing and thawing allowed for handling of DS did not specify when these may occur.

Section 3.2.S.7.2-Observations related to the post-approval stability protocol and stability commitment 6 included:

- Commitment was required to finalise the ongoing DS stability study, notify any OOS and place on stability 3 one DS batch each year submitting regular updates.

- The proposed post-approval stability protocol should be reviewed in line with any justification of the specification and stability.

- Detailed information on the post-approval stability study protocol was not provided.

- It was not demonstrated that tests in the stability protocol are capable of detecting degradation trends.

Section 3.2.S.7.3-Observations related to the stability data included:

- Stability data for commercial batches was not compared with the primary stability batches.

- It was not shown that DS stored at the specified conditions will yield acceptable purity and potency.

- More data was required on: specific impurities during storage; immeasurable results in photostability and 5 specified active substance batches being in compliance with specifications over defined period of time.

- Updated stability data should be submitted in due time. 
Table VI. (continued)

Category of deficiency

Absolute \% frequency of frequency of concern/specific concern (f) CTD module section

- The proposed hold time, wider end of shelf-life specifications and claimed shelf life were not supported by 3 sufficient or real data.

- There were unjustified omissions of certain tests from the stability protocol and unjustified choice of stability 3 tests.

- During storage, increased related proteins were observed; therefore, more stability studies at this storage condition should be provided.

- Details and data on transport of the DS were missing.

- Validation data for DS freezing step after filling was not provided.

- The duration of storing the undiluted DS batches after manufacture until stability studies was started was not indicated.

- More data required to verify the applicability of stability studies performed with a diluted DS solution to routine storage of DS.

- The shelf-life specification should be amended with some tests such as on: bio-burden; oxidised species; and on integrity of carbohydrate structures.

- Data showing that the stability data of specified active substance batches are in compliance to the specifications over a defined period was not provided.

Others-Not included in the above sections

Observations related to the adventitious agents evaluation included:

- Certificates of origin and CoA for the enzymes used during the cloning procedures and media used during the transfection and preparation of the primary seed lot were not provided.

- The safety of the DS with regard to viral and TSE risks was not documented.

- For in vitro testing of the cell banks/unprocessed bulk, it was unclear if haemagglutination was tested, and justified if not.

- It was not specified which bovine and porcine viruses can be detected by the assays and which methods are employed.

- The testing programme for the MCB and WCB included only one in vitro infectivity assay for retroviruses.

- Information on the laboratories where testing of future WCBs, testing of the unprocessed bulk and the virus 2 validation studies have and will be performed is missing.

- The linear flow rate for equilibration, loading, washing and elution steps was not given for the downscale model and the manufacturing process.

deficiencies reported were related to pharmaceutical development and stability.

Apart from observations on stability, which were common for both drug substance and drug product of chemical entities and biosimilar medicinal products alike, all the other deficiencies are not comparable. Perhaps, this should not come as a surprise since as expected, the development, production and control of chemical entities and biological products are fundamentally different, and hence, observed deficiencies will be expected to be different as well.

Similarly, as in the study by Borg et al. (15) for chemical entities, this study indicates that pharmaceutical companies could take note of the results obtained and presented here, to potentially avoid many of the observed concerns with respect to lack of information supplied by prospective applicants for both drug substance and drug product, if the guidance in the International Conference on Harmonisation (ICH) CTD $(16,17)$ and 'Notice to Applicants Vol. 2B' as published by the EC (18) are more rigorously followed.

Product-specific guidelines were issued for biosimilar monoclonal antibodies, recombinant follicle stimulation hormone, recombinant interferon beta, recombinant erythropoietin, low molecular weight heparins, recombinant interferon alpha, recombinant granulocyte-colony stimulating factor, somatropin and recombinant human insulin (19). From these, only the guideline for low molecular weight heparins contains quality considerations. However, CHMP's guideline on similar biological products (6) is up to date (recently updated in 2014), and it contains detailed information on quality considerations for all biosimilars as well as requirements for the choice of reference product, outlining some of the principles upon which comparability is established in the EU. Central to the approval of a biosimilar in the EU is a robust comparability programme with the reference medicinal product (20). The robust head-to-head comparison should demonstrate the similarity between biosimilar and reference product at all levels: physicochemical analyses, functional assays, in vitro or in vivo pharmacodynamics (PD) response and repeat dose toxicity study in non-clinical design (where needed) and usually inclusion of clinical trials covering pharmacokinetic (PK)/PD response, efficacy, safety and ability to induce an unwanted immune response. This mandatory comparability exercise is designed to reveal the degree of similarity to the reference medicinal product from a quality/safety and efficacy perspective. Minor differences between products' quality attributes may be acceptable if they are appropriately justified and are not clinically meaningful (21).

For all biosimilar products, prospective applicants are advised to approach the EMA and request advice from the Agency on the conduct of the various tests and trials necessary to demonstrate the quality, safety and efficacy of 
Table VII. 'Other Concerns' for Drug Product (DP)

Category of deficiency

Absolute \% frequency of

frequency concern/specific

(f) CTD module section

Module 3.2.P.1-Description and composition of the drug product

Section 3.2.P.1 - Observations related to the description and composition of the drug product (DP) included: 8

$8 /$ $495 \times 100=1.6 \%$

- There was lack of proper description of product type.

- DP presented as pyrogen-free without any supporting data.

- Volume overfills were unjustified in pharmaceutical development.

- Issues were observed in the quantitative composition tables such as unreferenced ingredients and undeclared 4 water nominal amount.

- Type of primary container and information on the stopper material including coating material was not submitted.

Module 3.2.P.2—Pharmaceutical development

Section 3.2.P.2.1-Observations related to components of the drug product (composition) included:

- There was unjustified amount of non-ionic surfactant used, above the critical micelle concentration level.

- Former primary packaging components responsible for the release of leachables were not indicated.

Section 3.2.P.2.2-Observations related to formulation development issues included:

- There were unjustified items: acceptance criteria for impurities; identity assay and glycerine test not included 7 in DOE response parameters; sub-visible particles tested at all low $\bullet$ conditions; use of accelerated conditions in studies supporting excipient levels used; presence of an alkali earth metal as heavy metal traces chelating agent; and concentration of non-ionic surfactant used.

- There was missing data demonstrating: indicated total fill volume sufficient to administer nominal dose.

- There was no indication if the proposed formulation has changed throughout clinical development of the DP.

- There was no rationale for differences in the amounts of carbohydrates and amino acids between the DP and RP.

- Results of tests on extractable volume and DS content after reconstitution of the DP with the solvent were not submitted.

Section 3.2.P.2.3-Observations related to manufacturing process development included:

- No results were provided from the non-metal equipment compatibility studies.

- Batches were produced at two separate sites, with one site showing issues relative to the other such as higher 5 impurities, statistically significant biological functionality test differences and biological potency plus higher analytical test variability included in the assay.

- It was unclear what the acceptable maximum manufacturing time is for the DP given that time and high temperatures during manufacturing had a significant impact on DP stability.

- Data was not submitted on: degradation products as a function of hold times and manufacturing conditions; 2 development and commercial batch analyses proving changes in lyophilisation conditions did not cause changes in product quality in terms of physicochemical and biochemical parameters and water content.

- Batch numbers used in clinical trials listed in quality documentation were different to those indicated in the clinical dossier.

Section 3.2.P.2.4-Observations related to container closure system (CCS) included: 6

- No data was submitted to: support the compatibility conclusion of the CCS and the DP; demonstrated 2 integrity of the CCS is maintained at the end of DP shelf life.

- CCS integrity was not investigated in a suitable manner and integrity test was not performed at incubation of 2 $30 \pm 2^{\circ} \mathrm{C}$ for 14 days.

- Use of uncoated rubber stoppers was unjustified.

- Storage conditions were not indicated for the proven storage time.

Section 3.2.P.2.5-Observations related to microbiological attributes included:

- There were inconsistencies in results of micro tests for preservative limits on pilot batches. According to EP 3 this should be with full-scale batches with lowest preservative limits, repeated with solvent reconstituted batches.

- Results were not provided for bacterial challenge test performed at incubation of $29-31{ }^{\circ} \mathrm{C}$ for 14 days.

Section 3.2.P.2.6-Observations related to compatibility:

- Compatibility with the formulation of different primary packaging components was not demonstrated and that these do not cause leaching.

Module 3.2.P.3-Manufacture

Section 3.2.P.3.1-Observations related to manufacturer included:

$35 /$

$495 \times 100=7 \%$

$2 / 35 \times 100=5.7 \%$

$31.4 \%$

11

(1)

(1)

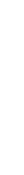

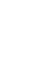

.

- An updated GMP certificate was not provided for alternative second

- An updated GMP certificate was not provided for alternative secondary packaging sites.

Section 3.2.P.3.2-Observations related to batch formula: 
Table VII. (continued)

Category of deficiency

Absolute

frequency

$(f)$

- The manufacturing formula was inaccurate and incomplete.

- There was no justification for: use of varying amounts of excipients and water during production; and use of 2 $\mathrm{pH}$ adjusters.

- The specification for extractable volume is unclear.

- The formula for calculation of the salts used is inconclusive.

Section 3.2.P.3.3-Observations related to description of manufacturing process and process controls included: 41

- It was unclear whether: several batches of DS can be pooled; process stream filters are single use; nitrogen 8 used in WFI flushing is $0.22 \mu \mathrm{m}$ membrane filtered.

- There was no justification why: final sterilising filter is not direct before filling and 2 sterilising filtration steps 7 are used; maximum filling time is higher than that provided in the results; temperature chosen for compounding DS with excipient solution; filtration of buffer formulation is required since bio-burden preand post-filtration is the same; $\mathrm{pH}$ limit was much wider than the recorded values; base filling on activity instead of mass is adopted.

- No information was given on: QC tests performed at different manufacturing facilities; microbiological 10 quality of starting materials; filter material and pre-filter pore size; lyophilisation conditions of the cycle used are not given and it is not confirmed that the lyophiliser is sterilised after each cycle.

- Missing or lack of data on: primary packaging material sterility; CoA of material used as solution contact 5 layer of bags used in production; thawing time for bulk active not substantiated by data on DS stability, biochemical and microbiological purity.

- Discrepancies were noted in: residual moisture between batches coming from separate sites; and filtration times.

- Maximum storage times for product, before and after labelling, were not defined based on stability data.

- The post-use integrity test for filters of the filter train sterilising the formulated bulk was not specified.

- There was inadequate description of the manufacturing process.

- Closure integrity of the bags used in production especially with respect to microbial contamination was not demonstrated.

- Flow sheet was not updated with: preparation of primary packaging material; hold times for processing steps; 3 and process controls for bio-burden and any additional controls.

- Retrospective adjustment of active concentration in bulk solution was not acceptable as a corrective action for IPC protein content.

Section 3.2.P.3.4-Observations related to controls of critical steps and intermediates included:

- There were unjustified items: limits for critical process controls; omission of IPC for fill weight; high rejection 5 rate at start of filling; sample size for IPC; high OOS in assay results of some lots.

- The following was not specified: number of unit tests for filling in process control; number of parallel runs 7 required to switch completely from old to new analytical methods; critical process parameters; IPC on identified critical steps; bio-burden determination in line with EP requirements.

- Specifications required update with respect to: reference to SOP of analytical method; impurity detected with 4 specified RP-HPLC; end of shelf-life limits.

- There was missing data supporting the use of only two tests for identity.

- There were no details of the microbial retention analytics for the DP sterile filtration concerning the type of medium used for the challenge.

- Test on fill height and particulate contamination were not added to the in-process controls.

- A specific batch had a clogged pre-filter during filtration failing the filter integrity test. Explanation on contributing factors (other than bio-burden which was within limits) was not provided.

Section 3.2.P.3.5-Observations related to process validation (including analytical tests) and evaluation included:

- A validated maximum batch size for commercial product is missing.

- Numerous media fill validation issues were identified including:

- Aseptic process not validated by media fill-generated data.

- Media fill validation not covering all product volumes and container types.

- Run not conducted with the same equipment used during the process.

- Details of the media fill conditions were not described.

- The nature and number of routine operations did not reflect all possible manipulations and conditions.

- Media qualification was not positively challenged supporting growth of low numbers of environmentally found microorganisms according to EP and using all species of aerobes, anaerobes and fungi.

- Data of at least three media fill runs were not submitted.

- For contamination rate less than $0.1 \%$, the identity of the contaminants was not made and source not investigated. 2

- There was no justification for: using a set of impurities in validating specificity of the HPLC methods used; 7 low moisture content of one validation batch; adopted endotoxin limits; several OOSs at certain steps for purity and content determinations for two commercial scale batches; upper $\mathrm{pH}$ limit for mixing and lyophilisation; use of sterile filter type in relation to its material compatibility, capacity, sorption and extraction of constituents.
$\%$ frequency of concern/specific CTD module section 
Table VII. (continued)

Category of deficiency

Absolute

frequency

(f)

3

- Incomplete validation data was submitted.

- Commitments required for: a reproducibility report prior to transferring method for identification to another 2 site; use of only dedicated tubing for DP to avoid contamination.

- The company did not provide DP batch consistency data on some parameters such as dimer and HMW species content.

- The following were not validated or qualified: the proposed hold periods for the unfiltered and filtered 2 formulation buffer; filling of vials with DP for protein losses during filtration at second site.

- There were no descriptions for: tests conducted on filter extractable; process and conditions for filter 7 sterilisation; validation procedures for the autoclaving of stoppers in their bags; thawing period and its conditions where required for tests; vial dehydrogenisation method used.

- Monitoring of bio-burden levels was not included in the process validation, especially to validate the holding period. 4

- Different criteria/parameters were used for validation of capping and crimping processes at different manufacturing sites.

- Hold time validation issues included:

- Hold times before and after filtration of DP bulk were not provided.

- Not carried out for final bulk except for aseptic filling.

- Hold time within lyophiliser chamber after cycle completion was not provided.

- Hold time between crimping and labelling was not provided.

- Few parameters were included in the hold time testing programme. Must include data on critical physicochemical parameters and microbiological purity.

- A correlation was not made between the purity of the DS batch used and the respective DP batch manufactured.

- The following issues related to validation in general were observed:

- Batches used were not representative of the intended batch ranges and volumes.

- Validated process parameters did not translate into appropriate limits for manufacturing processes.

- No validation data submitted supporting duration of sterilising filter.

- Homogeneity of the formulated bulk and filling not adequately validated.

- Validation data not submitted for all filling lines used.

- Validation should be done at both the compounding steps for formulation and after introduction of the DS.

- Mixing speed during compounding was not specified.

- B. subtilis was not accepted as an environmental germ and measures including monitoring were not taken to prevent future contamination.

- There was missing data for: temperature mapping of lyophiliser shelves; some IPC tests during filling stage. 2

- Duration time and/or holding times for all manufacturing steps not validated.

- The proposed maximum processing and holding times are not fully validated.

Others-Observations related to other unspecified areas under section P3:

- Observations related to validation of the shipping and transport chain included:

- Transportation validation from third countries not provided.

- Validation results for shipping of DP for packaging, labelling and release not provided.

- The validation protocol did not include land transportation.

- Criteria not set for batch rejection when validated shipping conditions are not maintained during transport.

- Report showing suitability of the CCS for the parenteral DP was not submitted.

- The proposed batch numbering system is unclear.

- The definitive range for commercial batch size was not stated based on the batch sizes used for process validation.

Module 3.2.P.4 - Control of excipients

Section 3.2.P.4.1-Observations related to specifications:

- Specifications and limits for starting materials, excipients, intermediates and WFI were not shown/confirmed 7 compliant with $\mathrm{Ph}$. Eur. respective monographs.

- WFI specifications issues were observed namely that:

- There was no confirmation that it was produced by distillation.

- The test for aluminium was missing.

- Test results for nitrate, heavy metals and endotoxins were not included.

- Nitrogen was not included in list of excipients.

- The following microbial parameters were not determined using pharmacopoeia procedures: microbiological 4 quality of the excipients; microbial and endotoxin limit acceptance criteria for all excipients used in the aseptic production of drug product.

Section 3.2.P.4.2-Observations related to analytical procedures: 3

- An outline of acceptance testing for excipients, some specified starting and intermediate materials that they are fully in line with $\mathrm{Ph}$. Eur. requirements was not available. 
Table VII. (continued)

Category of deficiency

Absolute

frequency

$(f)$

Section 3.2.P.4.3-Observations related to validation of analytical procedures:

- Validation data were not submitted for analytical testing methods of non-compendial substances.

Section 3.2.P.4.4-Observations related to justification of specifications:

- Some WFI specifications such as conductivity were not in line with Compendia limits and had missing tests. 2

- Material of animal origin was not assessed with respect to TSE risk and virus safety.

- Shelf-life and storage conditions of excipients were not documented.

Section 3.2.P.4.5-Observations related to excipients of human or animal origin:

Section 3.2.P.4.6-Observations related to novel excipients:

Module 3.2.P.5 - Control of drug product

Section 3.2.P.5.1-Observations related to specification(s) (name, dosage form) included:

- Release and/or shelf-life specifications did not include: test for bioactivity of DP; parameters for delivery 12 device; test for extractable volume; purity test for active forms; all detected impurities; deamidation.

- Specifications for control of some specified starting materials, buffers and excipients used were not tightened 2 enough.

- The level of impurities was not related to the amount of the main product and their limits were established 2 on the basis of actual results.

- Acceptance criteria and testing limits do not reflect DP historical batches achieved results.

- Information on the specifications, limits and on choice of tests was missing.

- Comparison of batch data of the common DS and DP specifications was not carried out and used as basis for proposed changes to the DP specifications.

- There was no justification for: differences in shelf-life specifications of diluent applied for vials and those on 7 stability; why one test method was used for identification of DS; upper limit of dimers and related substances of higher molecular mass.

Section 3.2.P.5.2-Observations related to analytical procedures included:

- The following tests were not in line with Ph. Eur. standards or requirements: test for visual inspection of 2 colour and turbidity; biological activity.

- The following developed test methods were not acceptable due to: isoelectric focusing not being robust 2 enough; content assay and impurities tests not discussed sufficiently.

- There were unjustified items: use of more than one test for purity; UV detection at a specific wavelength for 3 peptide mapping.

- Missing tests included physicochemical EP tests.

- Percentage/proportion of the batch to be checked during the manufacturing process was not specified.

- The use of only two tests for identity was not supported by data showing that the minor species resolved can be quantified.

- There was missing information for: sterility tests employed; when to use the included endotoxin test; some 6 test procedures; equipment used for sub-visible particles testing.

- The absence of interference of materials used in chromatography with the active monomer and dimer/ aggregate peaks should be demonstrated.

Section 3.2.P.5.3-Observations related to validation of analytical procedures included:

- The following validation tests were considered unacceptable due to: purity test did not include dimers and 16 HMW; validation in general lacked reproducibility reports; specificity part since not demonstrated using other molecules with similar molecular weight; impurities not included in LOD part; unsatisfactory detection of aggregates; chromatography validation unacceptable acceptance criteria of precision, accuracy, LOQ, LOD and range; determination of specific residue amounts in active substances; some validations in general were not in line with ICH Q2A and Q2B (13).

- There was no justification for: impurities chosen; differences in in vitro active neutralisation assay results 3 during method transfer; chosen organism during positive controls tests.

- The following tests were not in line with Ph. Eur. requirements: sterility tests and endotoxin limit at release 2 of DP.

- Test method for active oxidised species was not described in detail.

- The statistical evaluation of the bioassay was not provided.

- Validation reports for endotoxin testing, assay procedure and some manufacturing steps were missing or had 5 missing data.

Section 3.2.P.5.4-Observations related to batch analysis included:

- OOSs in specific amino acid content in batch analysis required further investigation. specified impurity; at least three manufacturing scale batches of finished product in line with revisions to the manufacturing process and product specifications.

- There were no explanations on: differences in related substances for DS and corresponding DP; no tests on 2 particulate contamination and on potency were done. 
Table VII. (continued)

Category of deficiency

Absolute

frequency

$(f)$

- CoAs for some specified batches, including those used in clinical trials, were not provided.

- There were missing data: batch analyses summaries; chromatograms of release-tested batches; full-scale 4 batch results of the diluent; batch analysis data using methods specified in release specifications.

- Updated batch analysis data and a full review of the specifications when more batches of formulated bulk 4 have been manufactured were required.

- Batches selected for analysis did not represent worst-case scenario batches.

Section 3.2.P.5.5-Observations related to characterisation of impurities:

- Since a high number of impurities, some not specified, originated from the solvent/chromatographic system and the excipients, a higher quality of starting materials was required.

Section 3.2.P.5.6-Observations related to justification of specification included:

- The specifications were not set on data derived from batches used in pre-clinical, clinical and stability data.

- There were unjustified items: end of shelf-life limits; endotoxin limits; pH specification limits; active salt 10 selection; omission of impurities in specifications; missing container closure test.

- The following were not considered in line with EP: particulate matter acceptance criteria; tests for 6 extractable volume; no quantitative test for carbohydrate heterogeneity; test on appearance.

- The following were missing from the specifications: preservative efficiency testing at the end of shelf life; 5 active content in reconstituted solution; product-related impurities in specifications considered as too wide; acceptance and extractable volume after reconstitution as well as uniformity of mass.

- The justification of specification was not satisfactory.

- The proposed specification range for the control of protein content was wider than the range of measured values.

- The LOD for the related proteins was not regarded as acceptable.

- Later re-evaluation based on more batch data should be submitted for purity release limits.

Module 3.2.P.6 - Control of drug product reference standards or materials

Section 3.2.P.6-Observations related to reference standards (RS) or materials included:

- The need for more than one RS should was not explained and their identification was not clear.

- No calibration of the internal standards was made against any international standard of the active.

- Characterisation of RS was incomplete, especially with regard to glycosylation, oxidised species and biological activity.

- Data discrepancies were noted in presented CoAs.

- The stability study was not acceptable because testing was too limited and no systematic testing schedule was 2 employed, with no information provided on the working standards used in the bioassay, including their preparation, characterisation, storage, monitoring of stability and acceptance criteria.

- Impact of differences in DS and DP formulation on use of substance formulated reference material for DP 2 analyses was not studied.

- There was missing information or issues with RS used such as: when and in which exact tests they could be 7 used; their antiviral bioassay; results of their identification, purity and content; and SOPs governing establishment of primary and/or secondary standards.

Module 3.2.P.7-Container closure system

Section 3.2.P.7-Observations related to the container closure system (CCS) included:

- Issues with quality control and testing parameters included:

- Coating composition of the stoppers was not given.

- Test instructions for routine testing of each packaging material component was outdated.

- CoAs for glass and rubber stoppers used were not provided.

- Routine test parameters and acceptance criteria for each CCS component were not listed.

- There was no justification for not including dose accuracy testing in batch release testing.

- Results on readability of instructions for use were missing.

- There were different transparency levels amongst submitted samples. One empty sample was also required 3 for assessment.

- The filling volume was not clearly indicated by a calibration mark.

- Primary packaging issues included:

- The quality of all primary packaging components materials were not defined, nor CE marked.

- Adequate in-house specification including all test parameters relevant to quality of the rubber stopper coating material along with acceptance criteria was not provided.

- Sterilisation of primary packaging components was not satisfactorily described.

- Manufacturers of primary packaging materials were not named.

- Secondary packaging components were not described and sufficiently protective.

- Compatibility of the coated stopper material with the DP was not demonstrated with product-specific 5 migration data on potential extractables. (10)

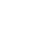

5

.

$\%$ frequency of concern/specific CTD module section

$1.6 \%$ 
Table VII. (continued)

Category of deficiency

Absolute

frequency

$(f)$

- Type and conditions of sterilisation/depyrogenation used for all CCS components including primary packaging, with validation data were not presented.

- Qualification report confirming accurate positioning of labels on primary packaging was not provided.

Module 3.2.P.8 - Stability

Section 3.2.P.8.1-Observations related to stability summary and conclusion included:

- The proposed shelf life was not supported by studies.

- SPC warnings were not as suggested from presented studies.

- Dilution for infusion in-use compatibility studies was not reported in detail and in-use studies tests were 2 missing.

- Summary tables with test results from stability studies conducted under accelerated and stressed conditions were not submitted.

- In stability studies, the samples were stored in an inverted position. The respective experiments were not assigned.

- Batches tested and used in validation were different with different lyophilization conditions. Therefore, it was not clear how representative all the batches were in terms of stability.

- There was no test method able to separate degradation products with different charges integrated in the ongoing stability programmes.

- The study design did not meet the requirements as proposed for in-use studies since the test programme was limited to assay.

Section 3.2.P.8.2-Observations related to post-approval stability protocol and stability commitment included: 12

- Routine stability protocol lacked some specifications and/or tests (e.g. particulate matter, extractable volume 2 and potency); and inclusion of ongoing stability studies/batches of DP produced with DS at the end of shelf life was not considered.

- Batches placed under stability study did not include maximum and minimum size of batches.

- The proposed post-approval stability study was unclear with respect to how many batches per strength are 2 annually put on stability testing.

- Proposed stability programme commitment did not fulfil the requirements of ICH Q5C (10).

- Closure integrity was not demonstrated after storage under accelerated conditions and at the end of the ongoing stability studies.

- Inclusion of the ongoing stability studies batches of DP produced with DS at the end of shelf life should be considered.

Section 3.2.P.8.3-Observations related to stability data included:

- The rate of HMWP formation was different for one DP batch raising concerns about manufacturing consistency.

- Proposed shelf life lacked supporting data and issues included: proposed shelf lives beyond real-time data; 8 end of shelf-life specifications not corresponding to available stability data; biological activity OOS at specific time points; and unproven declared shelf life.

- Effect of cartridge on the DP injection stability not shown in studies.

- Missing or insufficient data for: vacuum stress for container closure ingress testing; supporting storage out of 14 refrigeration; potency test performance during stability control; chromatograms from DP long-term, accelerated, and stressed stability studies; product-related impurities/substances tests; omission of aggregate formation in stability indicating parameters; sterility tests on preservative efficiency; temperature excursions at the end of the shelf.

- There were unjustified items: high particulate matter levels for some batches at some shelf-life points; for 11 sub-visible particles test; results for shake stability studies; conclusions for compatibility between diluted DP and any infusion solution and infusion bag material; different release and end of shelf-life acceptance limits; choice of stability tests and stability indicating parameters; test design and specifications for proposed in-use period; reduced set parameters applied during stability testing.

- Acceptance limits were not shown on stability graphs.

- Ongoing drug product stability studies for different manufacturing sites were not provided, confirming a similar stability.

- Information was not provided for: method description and validation for tests of N-glycans and deamidated 6 forms as used in the stability studies; purity issues observed during stability studies; reduced protein content for reconstituted in-use stability studies.

- Shipping validation was not carried out.

- Explanation was required for: reduced protein content in reconstituted in-use stability studies; differences in 2 related protein amounts between DS and DP batches used for stability studies.

- Photostability issues were encountered such as missing photostability data under normal conditions; data 6 showing that secondary packaging protects the UV-sensitive DS and that DS unrelated impurities did not increase with exposure to light and UV.
$\%$ frequency of concern/specific CTD module section

$17.8 \%$

$14.8 \%$

$13.6 \%$

$71.6 \%$ 
Table VII. (continued)

Category of deficiency
Absolute
frequency
$(f)$
$\%$ frequency of concern/specific
CTD module section

- There were missing tests during stability studies, like for example, volume in container, sterility and endotoxins.

- Shelf-life specifications had missing criteria or parameters.

- Updated stability report for the lyophilisates was not provided.

- Confirmation was required that ongoing stability studies were performed according to shelf-life specification on an annual basis.

- Leachables originating from the CCS have not been investigated.

- Batches placed on stability trials were not subjected to any updated shelf-life specification.

- There was no investigation on stability data of different types of primary packaging material suggesting that 4 the CCS can influence stability of the solvent in terms of preservative content.

Others-Not included in the above sections

Observations related to comparability:

- The comparison of the DPs showed a slight shift in the retention time of the different isoforms. Investigation was required supported by data obtained with a mix of both DPs, unless otherwise justified.

- Comparative analysis of aggregates was not completed on batches with the highest relative content of nonionic surfactant.

- Confirmation required that the optimised and validated peptide-mapping method was used for comparison of oxidised percentage in DPs.

- Analytical results supporting DS batches consistency with a reference preparation were lacking.

- Comparability of secondary and tertiary structure required confirmation at DP level as formulation and isolation steps may alter protein conformation.

the biosimilar medicinal product irrespective of which regulatory region they have been developed in (22-24). Furthermore and importantly, prospective applicants should note that the EMA may give a scientific opinion, in the context of cooperation with the World Health Organisation, for the evaluation of certain medicinal products for human use intended exclusively for markets outside the EU which do not exclude biosimilar medicinal products (25).

Interestingly, from the 22 MAAs reviewed in this study, granting of a marketing authorisation was refused in only one application, with the other applications being satisfactorily and positively concluded after the applicant addressed the raised concerns and objections at day 120 by the CHMP. To the best of our knowledge, this is the first study carried out on quality deficiencies raised during the scientific evaluation of biosimilar MAAs by the CHMP since Regulation 726/2004/EC became effective. Our intention has been to increase transparency to facilitate development and reduce unnecessary and potentially avoidable delays in the registration process. This could ultimately result in increased access to safe and effective biological medicines reaching the EU market which will be of benefit to patients.

\section{CONCLUSION}

The main objective of this study was to provide a general understanding and a comprehensive list of commonly encountered regulatory issues during development, production and control of biosimilars in a way which can help sponsors and manufacturers of future biosimilar products to anticipate and avoid common pitfalls. It could also help stakeholders understand the requirements for compilation of better quality dossiers to be submitted in support for biosimilar medicinal products' marketing authorisation applications. The information provided indicates that general aspects of biotechnology product development can be improved, not just those factors relating to the demonstration of biosimilarity. Thus, as a result, this study will help pharmaceutical developers and manufacturers in reducing unnecessary and avoidable delays in the authorisation of these products to the benefit of patients.

The study can be of interest not only to pharmaceutical developers and manufacturers of biosimilars, but equally of interest to regulators as it provides a comprehensive compilation of consistent issues encountered by manufacturers in biosimilars' production and in the quality part of their submitted dossiers for a marketing authorisation. The findings of this study could facilitate future guidance and technical assistance for manufacturers submitting dossiers for marketing authorisations of biosimilars at an EU level.

Funding information The research work disclosed in this study was funded by the Malta Government Scholarship Scheme.

\section{COMPLIANCE WITH ETHICAL STANDARDS}

Conflict of Interest The views expressed in this article are the personal views of the authors and may not be used or quoted as being made on behalf of, or reflecting the position of, any national competent authority, any university, the European Medicines Agency or one of its committees or working parties. The authors declare no Conflict of Interest. 


\section{REFERENCES}

1. European Commission. Regulation (EC) No $726 / 2004$ of the European Parliament and of the Council of 31 March 2004 laying down community procedures for the authorisation and supervision of medicinal products for human and veterinary use and establishing a European Medicines Agency. Official Journal of the European Communities. 2004; L 136:1-33.

2. Committee for Medicinal Products for Human Use (CHMP). Guideline on the common technical document for the registration of pharmaceuticals for human use: organisation of common technical document. European Medicines Agency. 2004. http:// www.ema.europa.eu/docs/en_GB/document_library/ Scientific_guideline/2009/09/WC500002721.pdf. Accessed 12 Apr 2017.

3. European Medicines Agency (EMA). Committee for Medicinal Products for Human Use (CHMP).http://www.ema.europa.eu/ e ma/index.jsp ? curl=pages/about_us/general/ general_content_000094.jsp\&mid=WC0b01ac0580028c79. Accessed 17 Aug 2017.

4. Chow SC, Wang J, Endrenyi L, Lachenbruch PA. Scientific considerations for assessing biosimilar products. Stat Med. 2013;32(3):370-81.

5. Karalis VD. Bioequivalence to biosimilarity: the rise of a novel regulatory framework. Drug Res. 2016;66(1):1-6.

6. Committee for Medicinal Products for Human Use (CHMP). Guideline on similar biological medicinal products. European Medicines Agency. 2014. http://www.ema.europa.eu/docs/ en_GB/document_library/Scientific_guideline/2014/10/ WC500176768.pdf. Accessed 14 May 2017.

7. Committee for Medicinal Products for Human Use (CHMP). Guideline on similar biological medicinal products containing biotechnology-derived proteins as active substance: quality issues. European Medicines Agency. 2012. http://www.ema.europa.eu/ docs/en_GB/document_library/Scientific_guideline/2014/06/ WC500167838.pdf. Accessed 14 May 2017.

8. European Medicines Agency. European Public Assessment Reports for Biosimilars. http://www.ema.europa.eu/ema/ index.jsp?curl=pages \%2Fmedicines $\% 2$ Flanding $\% 2$ Fepar_search.jsp\&mid=WC0b01ac058001d124\&searchTab=searchByAuthType \&alreadyLoaded=true \&isNewQuery=true \&status $=$ Authorised\&keyword=Enter+keywords\&searchType=name\&taxonomyPath $=\&$ treeNumber $=\&$ searchGenericType $=$ biosimilars \& ge nericsKeywordSearch. Accessed 14 May 2017.

9. International Conference on Harmonisation (ICH). The common technical document for the registration of pharmaceuticals for human use-quality M4Q (R1). 2002. Available from: URL: http://www.ich.org/fileadmin/Public_Web_Site/ICH_Products/ CTD/M4_R1_Quality/M4Q R1_pdf. Accessed 15 May 2017.

10. International Conference on Harmonisation of Technical Requirements for Registration of Pharmaceuticals for Human Use. Quality of biotechnological products: stability testing of biotechnological/ biological products - Q5C. 1995. https://www.ich.org/fileadmin/ Public_Web_Site/ICH_Products/Guidelines/Quality/Q5C/Step4/ Q5C_Guideline.pdf. Accessed 14May 2017.

11. International Conference on Harmonisation of Technical Requirements for Registration of Pharmaceuticals for Human Use. Specifications: test procedures and acceptance criteria for biotechnological/biological products - Q6B. 1999. https:// www.ich.org/fileadmin/Public_Web_Site/ICH_Products/Guidelines/Quality/Q6B/Step4/Q6B_Guideline.pdf. Accessed 14 May 2017.

12. International Conference on Harmonisation of Technical Requirements for Registration of Pharmaceuticals for Human Use. Specifications: test procedures and acceptance criteria for new drug substances and new drug products: chemical substances Q6A. 1999. http://www.ich.org/search.html?id=192\&q=6A. Accessed 14 May 2017.
13. International Conference on Harmonisation of Technical Requirements for Registration of Pharmaceuticals for Human Use. Validation of analytical procedures: text and methodology Q2(R1). 2005. https://www.ich.org/fileadmin/Public_Web_Site/ ICH_Products/Guidelines/Quality/Q2_R1/Step4/ Q2_R1_Guideline.pdf. Accessed 14 May 2017.

14. Declerck PJ. Biologicals and biosimilars: a review of the science and its implications. Generics Biosimilars Initiative J. 2012;1(1):13-6.

15. Borg JJ, Robert JL, Wade G, Aislaitner G, Pirozynski M, Abadie $\mathrm{E}$, et al. Where is industry getting it wrong? A review of quality concerns raised at day 120 by the Committee for Medicinal Products for Human Use during European centralised marketing authorisation submissions for chemical entity medicinal products. J Pharm Sci. 2009;12(2):181-98.

16. International Conference on Harmonisation of Technical Requirements for Registration of Pharmaceuticals for Human Use. Organisation of the common technical document for registration of pharmaceuticals for human use - M4(R3). 2009. http:// www.ich.org/fileadmin/Public_Web_Site/ICH_Products/CTD/ M4_R4_Organisation/M4_R4_Granularity_Document.pdf. Accessed 14 May 2017.

17. International Conference on Harmonisation of Technical Requirements for Registration of Pharmaceuticals for Human Use. Pharmaceutical development - Q8(R2)2. 2009. https:// www.ich.org/fileadmin/Public_Web_Site/ICH_Products/Guidelines/Quality/Q8_R1/Step4/Q8_R2_Guideline.pdf. Accessed 14 May 2017.

18. European Commission. Notice to applicants volume 2B: the rules governing medicinal products in the European Union. 2006. http://ec.europa.eu/health//sites/health/files/files/eudralex/ vol-2/b/update_200805/ctd_05-2008_en.pdf. Accessed 14 May 2017.

19. European Medicines Agency (EMA). Product Specific Guidelines Available from: URL: http://www.ema.europa.eu/ema/ index.js p ? curl=pages/regulation/general/ general_content_000408.jsp\#Product-specific biosimilar guidelines. Accessed 15 May 2017.

20. Daller J. Biosimilars: a consideration of the regulations in the United States and European union. Regul Toxicol Pharmacol. 2016;76:199-208.

21. Markus R, Liu J, Ramchandani M, Landa D, Born T, Kaur P. Developing the totality of evidence for biosimilars: regulatory considerations and building confidence for the healthcare community. BioDrugs. 2017;31(3):175-87.

22. United States Food and Drug Administration. Scientific considerations in demonstrating biosimilarity to a reference product: Guidance to industry. 2015. http://www.fda.gov/downloads/ Drugs/GuidanceComplianceRegulatoryInformation/Guidances/ UCM291128.pdf. Accessed 15 May 2017.

23. United States Food and Drug Administration. Quality considerations in demonstrating biosimilarity of a therapeutic protein product to a reference product: guidance to industry. 2015. http://www.fda.gov/ downloads/Drugs/GuidanceComplianceRegulatoryInformation/ Guidances/UCM291134.pdf. Accessed 15 May 2017.

24. United States Food and Drug Administration. Formal meetings between the FDA and biosimilar biological product sponsors or applicants: guidance for industry. 2015. http://www.fda.gov/ downloads/Drugs/GuidanceComplianceRegulatoryInformation/ Guidances/UCM345649.pdf. Accessed 15 May 2017.

25. European Medicines Agency. Guideline on procedural aspects regarding a CHMP scientific opinion in the context of cooperation with the World Health Organisation (WHO) for the evaluation of medicinal products intended exclusively for markets outside the Community. 2005. http:// www.ema.europa.eu/docs/en_GB/document_library/ Scientific_guideline/2009/09/WC500003883.pdf. Accessed on 17th Aug 2017. 